\title{
NEW ASPECTS OF FRECKLE FORMATION DURING SINGLE CRYSTAL SOLIDIFICATION OF CMSX-4
}

\author{
R. Schadt, I. Wagner, J. Preuhs*, P.R. Sahm \\ Gießerei-Institut, RWTH Aachen, Intzestr. 5, 52056 Aachen, Germany \\ *DONCASTERS Precision Castings - Bochum GmbH, Bessemer Str. 80, 44793 Bochum, Germany
}

\begin{abstract}
Freckle formation is a long time investigated but in its high complexity still not completely understood phenomenon. Some new aspects of this phenomenon especially concerning freckle formation inside single crystal castings and the simulation of freckling are alighted. A simple and near process criterion for the prediction of freckle threatened areas in single crystal components has been developed. The investigations reveal that there are still a lot of open questions concerning where freckle formation exactly takes place. Nevertheless the results presented offer new starting points concerning solutions and may inspire to intensify the research activities on this interesting phenomenon.
\end{abstract}

\section{Introduction}

Freckles are presently one of the main defects encountered in advanced directional solidification (DS) and single crystal (SC) casting technology of superalloys. They appear as long chains of equiaxed grains preferentially at the component's surface. A reasonable explanation of freckle formation is thermosolutal convection driven by a density inversion in the mushy zone. This is caused by alloy specific segregations, which is indicated by a noticeable shift of the freckles grain composition towards the alloy's eutectic composition. The convection evolution is influenced by the component geometry as well as by alloy composition and primary process parameters as temperature gradient $G$ and solidification velocity $v[1-3]$. Today the most complete criterion available seems to be the Rayleigh criterion. It combines the influences of alloy composition and process parameters but does not take into account component geometry.
Sarazin and Hellawell [4] suggested to characterize fluid flow associated with freckle formation by using the Rayleigh number as follows:

$$
\text { Freckling when: } \quad R a=\frac{g \frac{d \rho}{d z}}{\frac{\eta D_{t}}{h^{4}}}>R a^{*}
$$

where the parameter $h$ is a characteristic linear dimension of the system linked to the dendritic array in the mushy zone $[3,4]$ :

$$
h^{4}=\lambda_{1}{ }^{4} \quad \text { or } \quad h^{4}=K \lambda_{1}{ }^{2} \text {. }
$$

Auburtin et al. [5] investigated the influence of the growth front angle on the freckle formation and modified the Rayleigh criterion. However this highly sophisticated criterion requires enormous efforts in measuring alloy specific thermophysical and thermochemical data.

In order to predict freckle threatened areas in complicated DSor SC-components it is necessary to develop a criterion, which takes into account process parameters as well as geometrical effects but does not depend on the complete knowledge of alloys' chemistry.

Such an accurate criterion enables the production of specific freckle tainted specimens for an investigation of their lifetime relevant effects on DS- and SC-components. 


\section{Experimental}

The SC casting experiments were carried out in an industrial sized vacuum Bridgman furnace where solidification conditions are the same as in those furnaces used for production of DS/SC parts. The chosen solidification parameters were similar to those normally used in the production process. Withdrawal velocities from $v=0.5 \mathrm{~mm} / \mathrm{min}$ up to $3.0 \mathrm{~mm} / \mathrm{min}$ and heater temperatures $\mathrm{T}=1500^{\circ} \mathrm{C}$ were applied. A round copper chill plate of $150 \mathrm{~mm}$ diameter was used for the cyclic clusters. Ceramic molds $\left(\mathrm{Al}_{2} \mathrm{O}_{3} / \mathrm{SiO}_{2}\right.$ based) were manufactured by standard investment process. Up to 20 thermocouples could exactly be positioned in the different specimens. The alloy CMSX-4 [6] was chosen for the investigations because due to its segregation behavior it is a typical freckle prone alloy. Furthermore there is great interest in widening the application range of CMSX-4 to large IGT-blades. The composition of the alloy is given in Table $I$.

Table I: Composition of the investigated alloy CMSX-4 (wt\%) [6].

\begin{tabular}{cccccccccc}
\hline $\mathrm{Cr}$ & $\mathrm{Co}$ & $\mathrm{Mo}$ & $\mathrm{W}$ & $\mathrm{Ta}$ & $\mathrm{Re}$ & $\mathrm{Al}$ & $\mathrm{Ti}$ & $\mathrm{Hf}$ & $\mathrm{Ni}$ \\
\hline 6.5 & 9 & 0.6 & 6 & 6.5 & 3 & 5.6 & 1.0 & 0.1 & bal \\
\hline
\end{tabular}

Several cylindrical geometries with constant or varying diameter were examined, Figure1. Freckle chains were characterized by Energy-Dispersive-X-Ray (EDX) and by Electron-BackScattering-Diffraction (EBSD). Composition and orientation distribution of the freckle chains in comparison to their counterparts in the single crystal matrix were examined in detail.

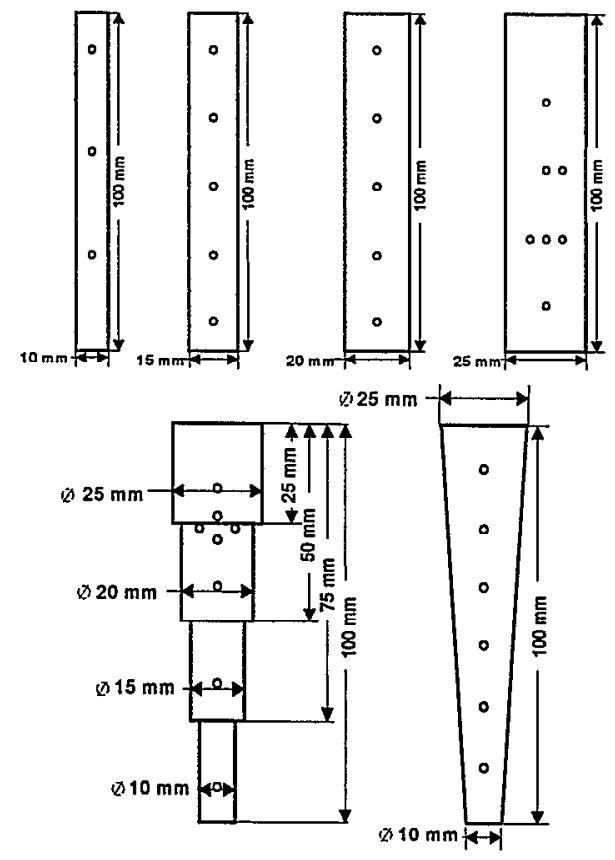

Figure 1: Outline of the investigated simple specimen geometries. The samples were solidified in clusters which consist of either four specimens with constant or four specimens with varying diameter. The circles indicate the position of the thermocouples.
All FEM-simulations were performed with the in-house program CASTS [7-10]. Simulations of grain growth were done with a cellular automaton (CA) algorithm [11].

\section{Results and discussion}

\section{$\underline{G^{*} v \text {-criterion }}$}

Based on temperature measurements with thermocouples exactly positioned in a ceramic shell mold a new, more simple and near application criterion for freckle formation has been developed. Late experiments prove, that the freckle chains do not always grow along the direction of the primary dendritic solidification front representing the crystallographic orientation, Figure 2. The comparison with FEM-simulations shows that the curved freckle chain which is visible on the surface of the turbine blade follows nearly exactly the curvature of the solidus line. According to the fact that freckle formation takes place during the last period of solidification, the measured velocity of the solidus isotherm and the temperature gradient just in front of this isotherm were used to generate a $G^{*} v$-criterion suitable for practical use. It takes into account the varying local solidification conditions during the Bridgman process. The results of these experimental investigations are shown in Figure 3. It is revealed that freckling only occurs below the critical threshold value $G^{*} v=0.14 \mathrm{~K} / \mathrm{s}$. In a transitional area, which cannot be determined exactly, most likely freckling mainly depends on geometrical influences.

Based on the temperature field calculations the experimental $G^{*} v$-criterion could be transferred to the simulation. It was implemented as a post processing tool. Freckle threatened and certainly freckle free areas can accurately be predicted, Figure 4. This criterion cannot predict where freckling actually will take place. A new approach in the calculation of freckle formation using coupled nonequilibrium FEM-methods and cellular automaton calculations is part of the ongoing investigation. First results of a 3D-CA coupled with equilibrium FEMcalculations are presented later.

\section{Expanding specimens}

The microstructure analysis of the stepwise and continuous expanding specimens reveals no tendency for freckle formation inside the specimens. Even if a freckle was found at the surface of a specimen in front of a step the freckle chain could not penetrate the castings inside more than a few millimeters, Figure 5 and Figure 7.

Experiments, in which specimens with stepwise and continuous expansion of cross-section were cast together in one cluster under the same process conditions show the same tendency. The specimen with continuously increasing cross-section shows one freckle chain starting at a diameter of $12.5 \mathrm{~mm}$ and growing along the surface of the whole specimen. The stepwise expanding specimen contains surface freckle chains at each diameter, but these chains always stop at a foot of a new step. 

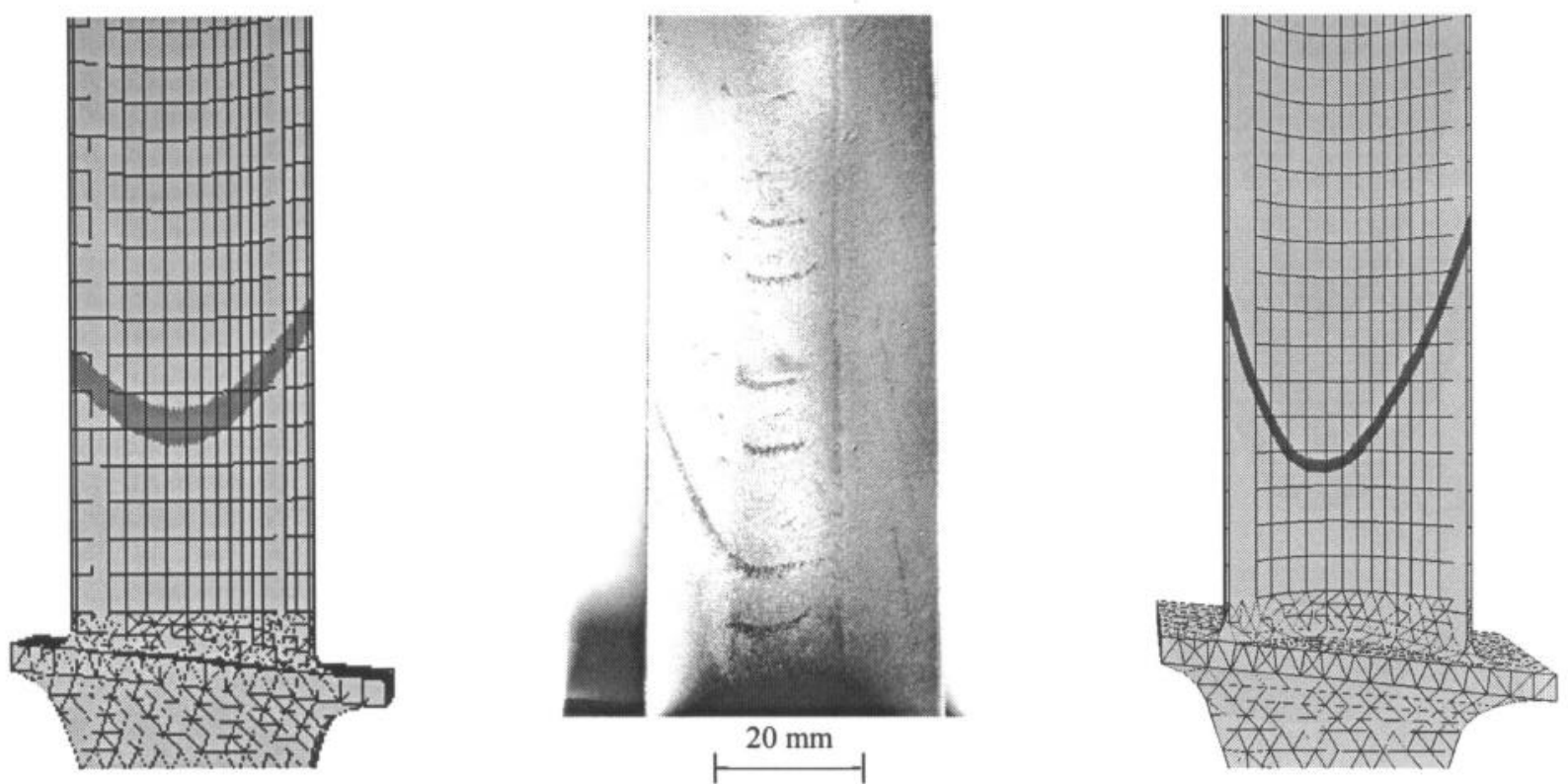

Figure 2: Calculated liquidus isotherm (left picture), turbine blade with extended freckling and calculated solidus isotherm (right picture) of an aero-engine turbine blade. The curvature of the freckle chains on the turbine blade seems to follow the solidus more than the liquidus line. The intermittent freckling structure occurs due to the non steady state conditions with locally varying growth velocities. The vertical temperature gradient $G_{v}$ was slightly decreased, the lateral gradient $G_{l}$ increased. Due to the torsion and the varying wall thickness not all freckle chains rises upward at the outer edges of the blade. Additionally should be remarked, that the liquidus and solidus isotherms have not the same propagation velocity. Simulations were performed with the FEM-program CASTS.

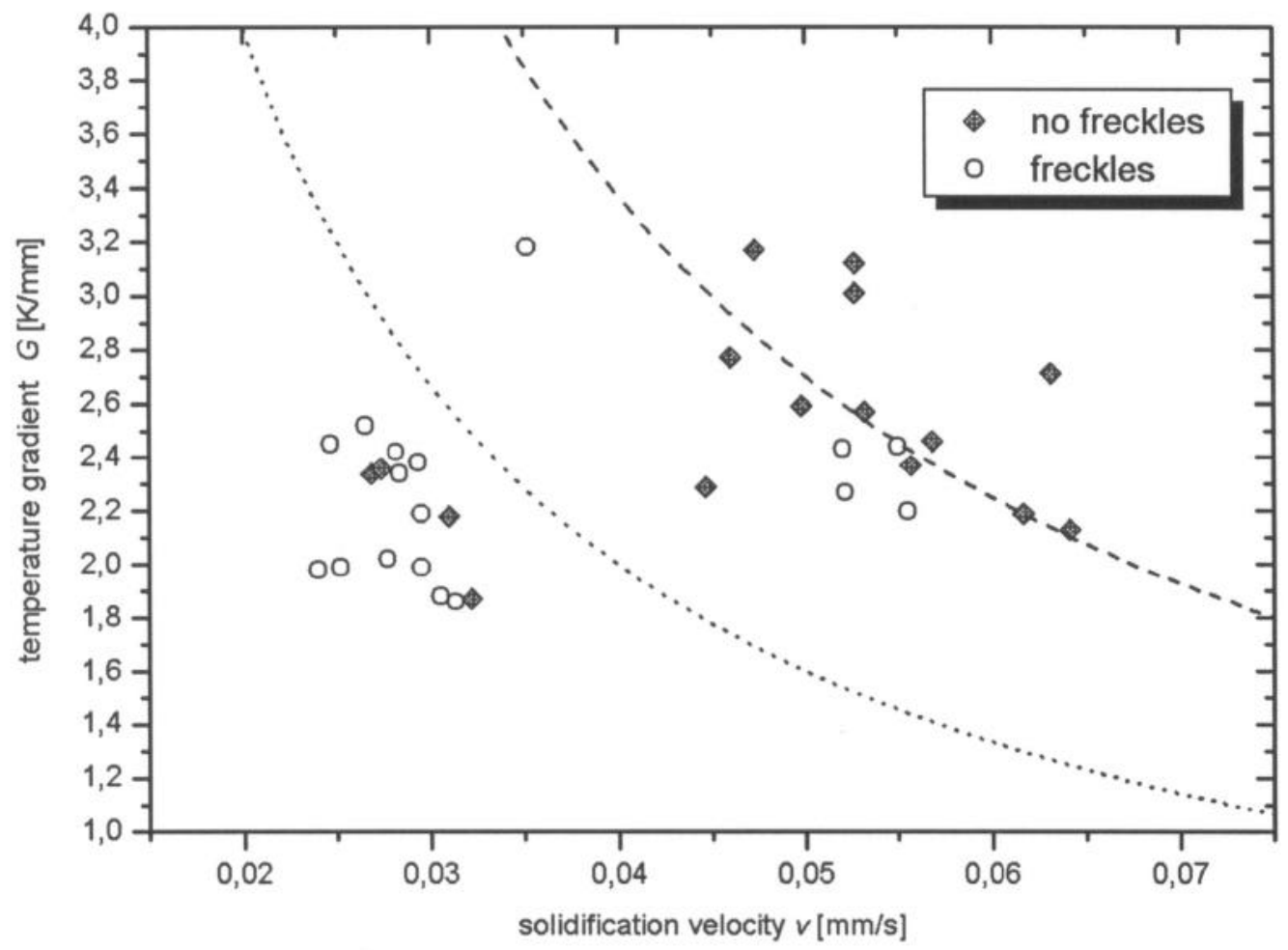

Figure 3: Freckle tendency of the Ni based superalloy CMSX-4. Above the critical threshold value $G^{*} v=0.14 \mathrm{~K} / \mathrm{s}$ (dashed line) no freckling occurs. In the transitional area (between the dashed and the dotted line) freckling seems to depend mainly on geometrical influences. 
After that a new chain starts a few millimeters above the step at a different position according to the cluster's centerline. This indicates that the newly arising freckle chain is not correlated to the one that stopped at the step's foot. It does only occur because the conditions at the surface are prone for freckling, Figure 6.
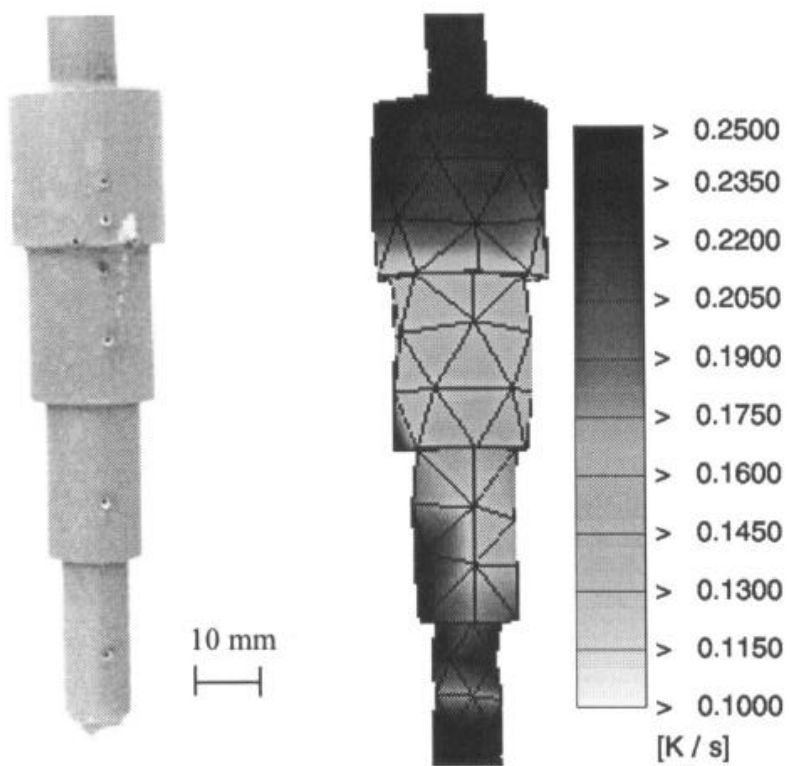

Figure 4: Comparison of a freckle tainted specimen with the simulated situation. The freckle chain appears in the region of the lowest calculated $G^{*} v$-value. The lack of symmetry in the simulation is due to the inhomogeneous radiation conditions of the cluster configuration.

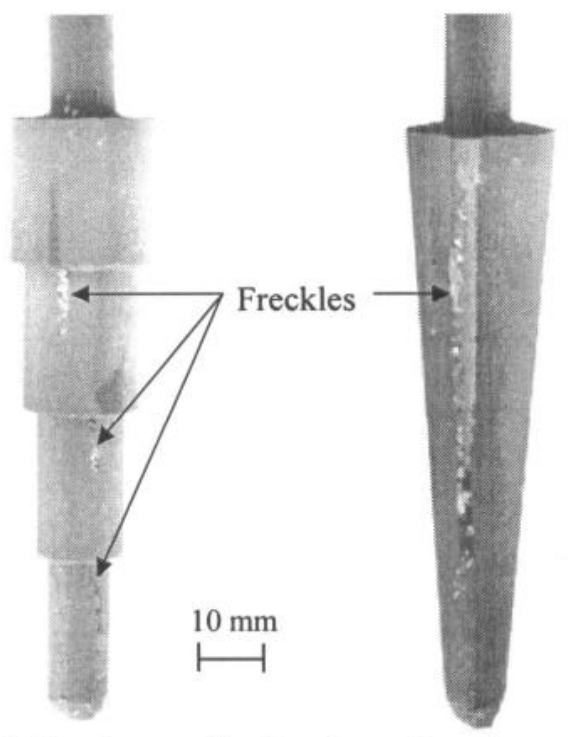

Figure 6: Specimens with stepwise and continuous increasing diameter. Whereas the step geometry shows different freckle chains at the foot of each step, the continuous expanding specimen contains one single freckle chain, which grew from a diameter of $12.5 \mathrm{~mm}$ to the top.

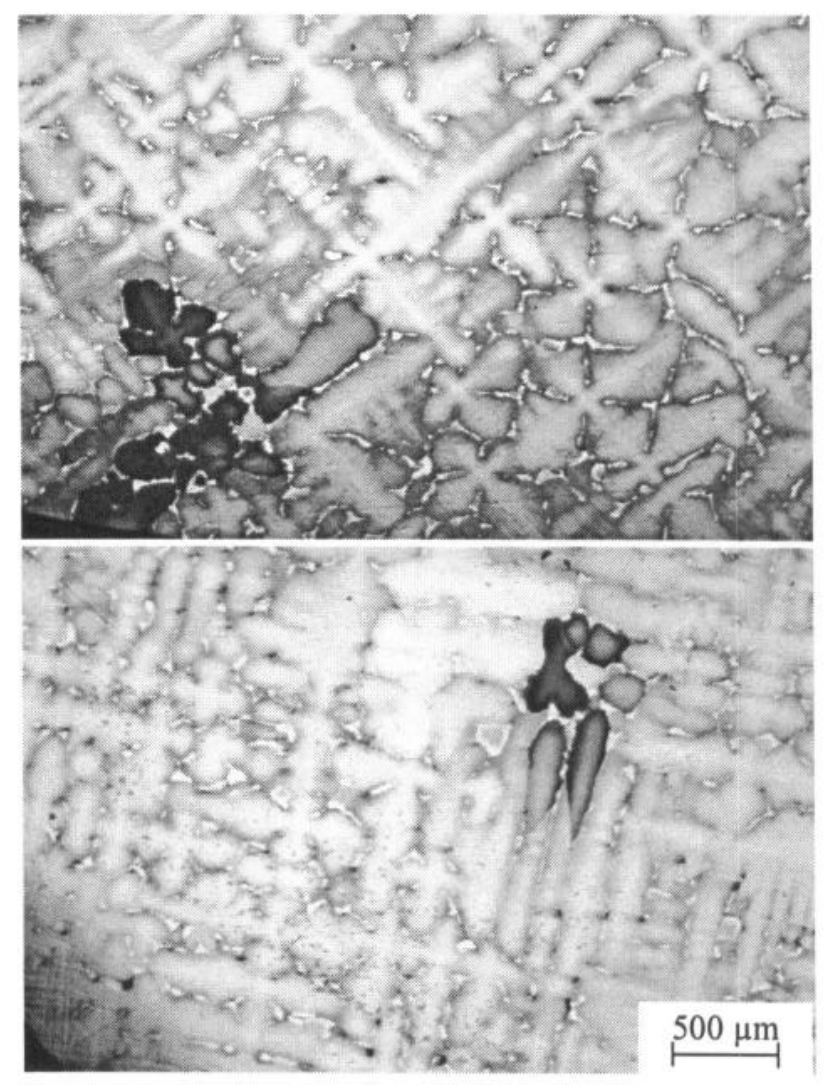

Figure 5: Microstructure of a stepwise expanding specimen. A grain of a freckle chain is visible at the surface of the specimen in a transversal micrograph just below the step. $400 \mu \mathrm{m}$ after the expansion only a small grain of the freckle chain is still visible in the single crystal matrix.

Additional investigations were performed on the question, if segregation channels, which are predicted by some authors [12-14] can be detected above the edge of these step specimens even if there was no freckling detected. 2D-simulations predict the formation of convection channels (plumes) in expanding domains [13]. Therefore freckle free specimens were investigated by EDX-analysis, Figure 8. Because the channels were expected to be in the dimension of dendrite axe spacing, a scan distance of $100 \mu \mathrm{m}$ was chosen. The measured line scans show no significant concentration decrease or increase of any element in the area above the edge of the step, Figure 9 and Figure 10. A few microns after the cross-sectional transition the distribution seems to be disturbed in the expanded area, Figure 9. But the distribution flattens with the distance from the edge, Figure 10. The high variations in the concentration of neighboring spots are due to the interdendritic segregations which are opposite for $\mathrm{Ti}$ and $\mathrm{Ta}$ on the one and $\mathrm{Re}$ and $\mathrm{W}$ on the other hand. 


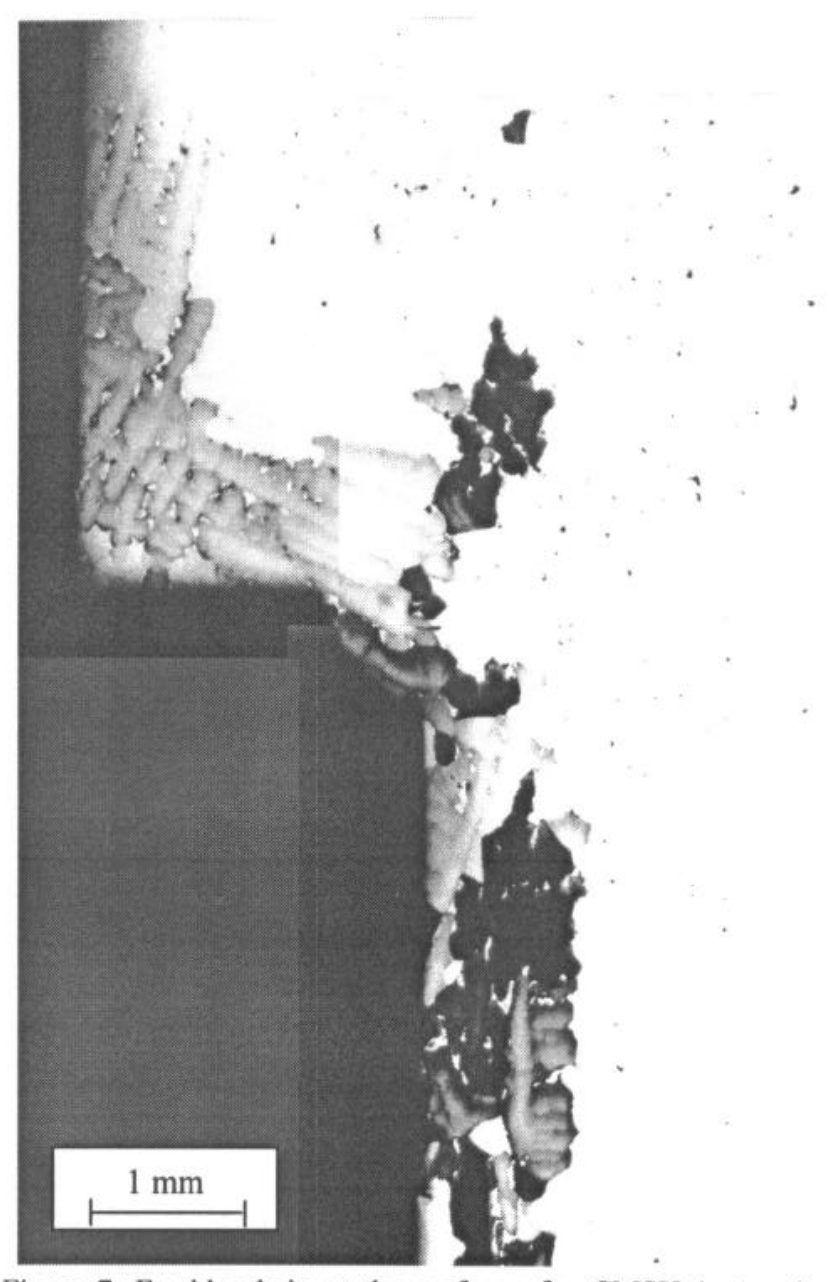

Figure 7: Freckle chain at the surface of a CMSX-4 stepwise expanding specimen. The freckle chain ends at the edge of the step probably due to a disturbance of the convection flow by the expansion. At the surface of the expanded part a slightly disoriented grain develops.

A characterization of the freckle chain in Figure 7 with EBSDanalysis gives an orientation distribution which indicates that there are no significant correlations between the single grains in the freckle chain, Figure 11. The polar plot shows the position of the [100]-orientations of the SC-matrix and the other measured grains. These orientations seem to be distributed quite coincidentally, but the high disorder is not only due to the different orientations in the chain but also to some fuzziness which is typical for the EBSD-analysis. The best focus is given in the center of the investigated specimen and the more the beam gets out of the center the more fuzzy the reflexes are. The matrix reveals a nearly perfect [100]-orientation, which is indicated by the dots in the polar plot.

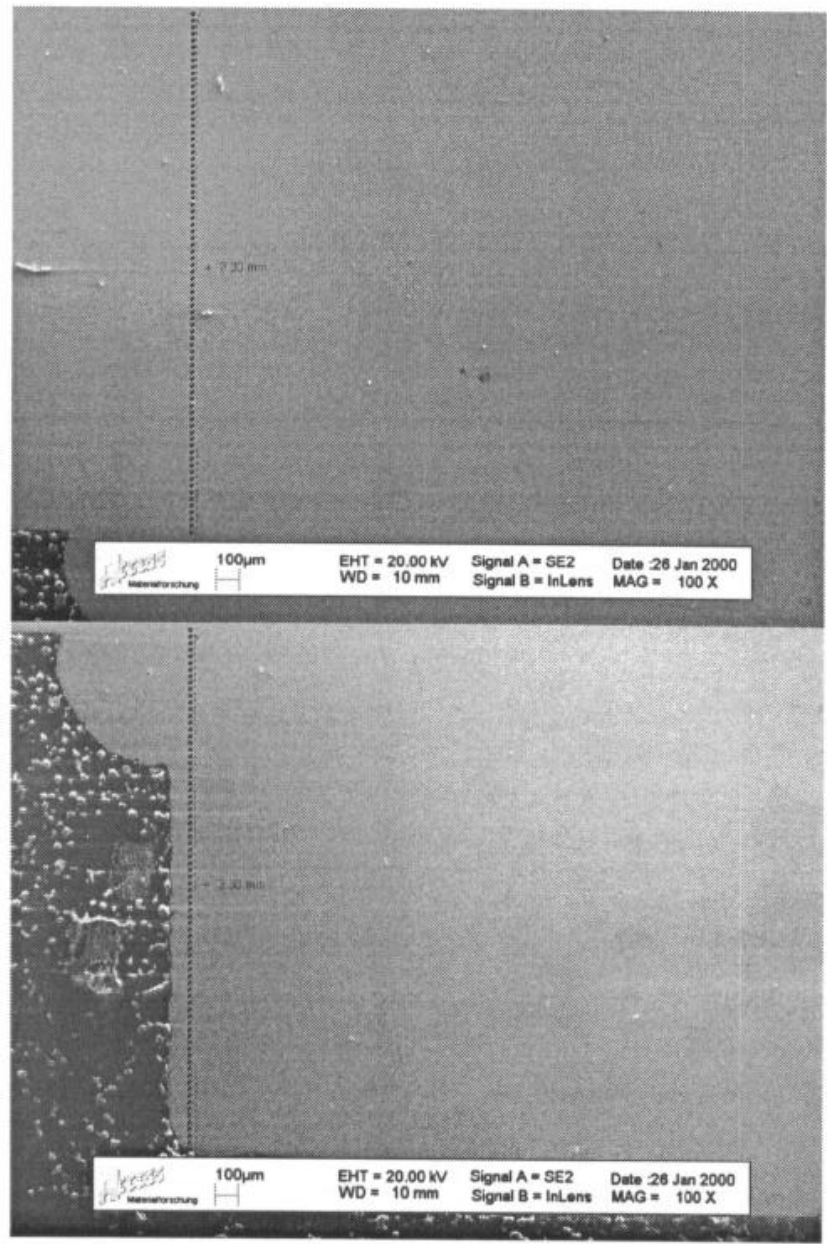

Figure 8. Expanding domain specimen investigated by EDX. The dashed line shows the path of the line scan. The distance between the measure spots was $100 \mu \mathrm{m}$, so that there were 46 measure points for each line scan. Two more line scans were performed at a distance of $7.5 \mathrm{~mm}$ and $12.5 \mathrm{~mm}$ from the edge.

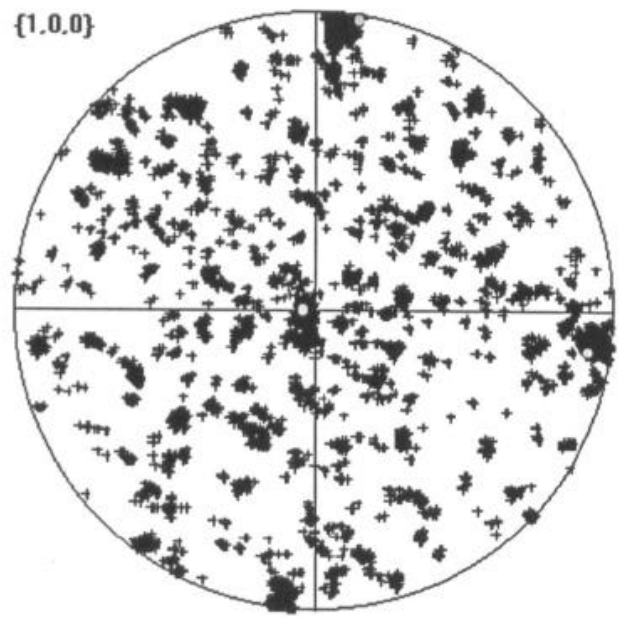

Figure 11: Polar plot of the measured [100]-directions of the SC-matrix (dots) and some freckle chain grains. 

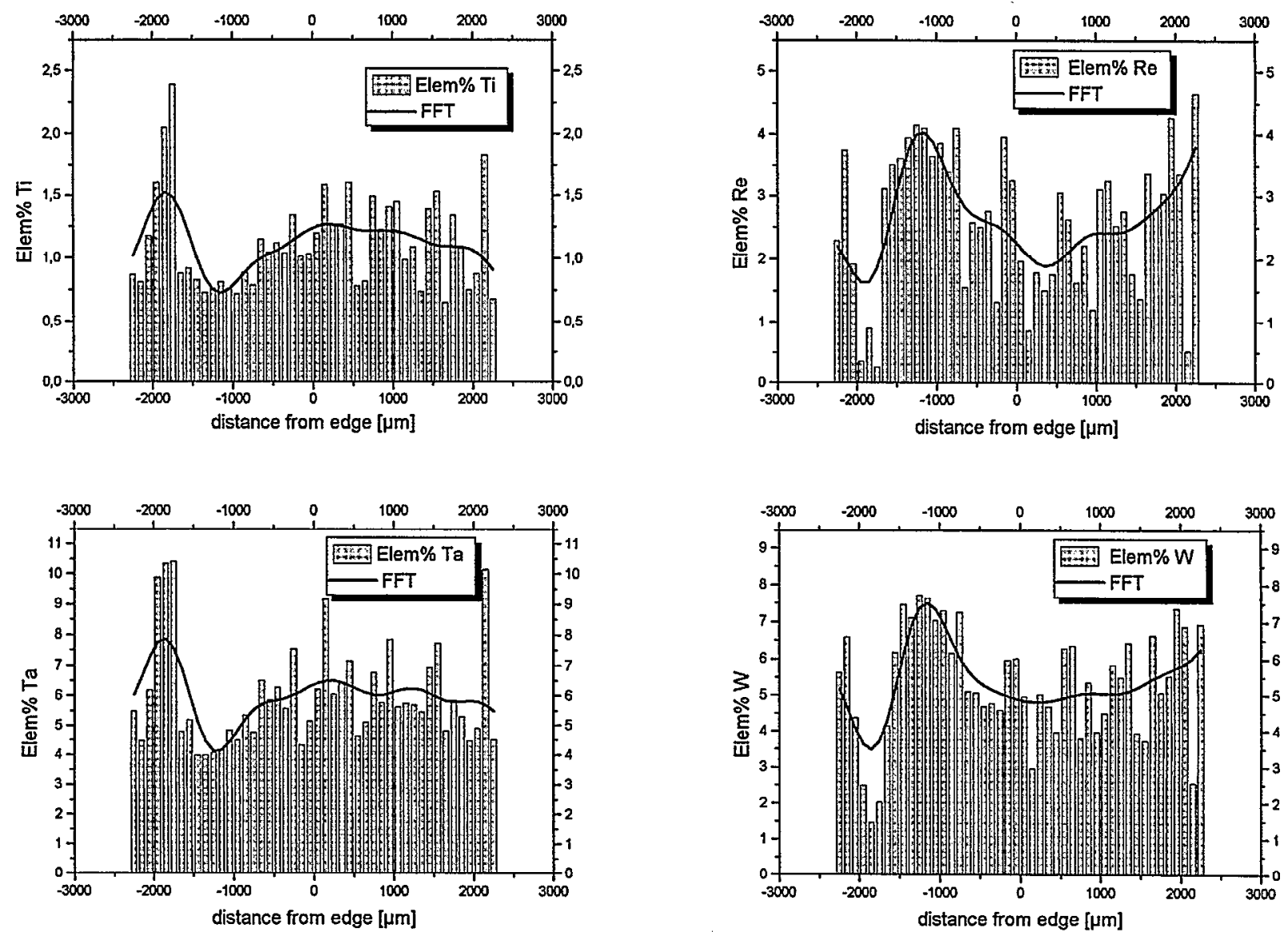

Figure 9: The EDX-line scan just above the edge of the expanding domain shows no significant concentration variation. It seems that the element distribution in the outer part of the domain (negative distance) is disturbed by the expansion. The columns display the concentration measured at each spot, whereas the lines represent an integral Fourier smoothing function term.
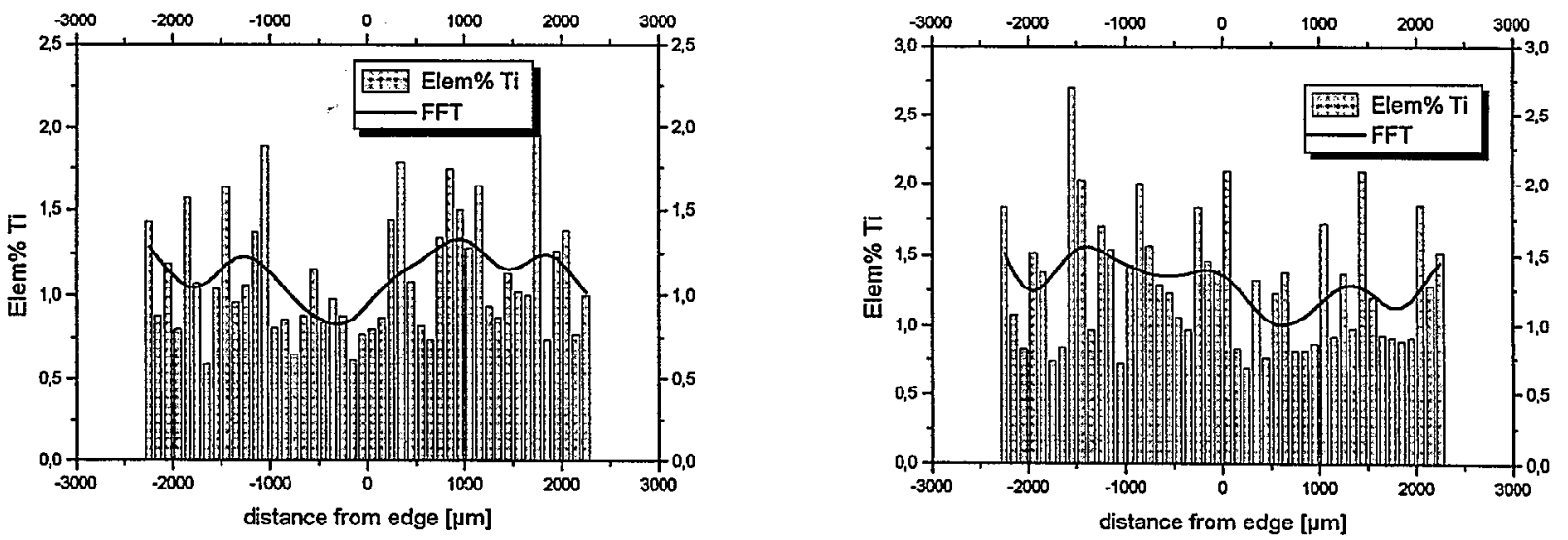

Figure 10: Titanium distribution $7.5 \mathrm{~mm}$ (left) and $12.5 \mathrm{~mm}$ (right) above the edge. The distribution seems to flatten with the distance above the edge. The high variations between neighboring spots are due to the interdendritic segregations. 


\section{$\underline{\text { Grain structure simulation }}$}

The simulation of the grain structure formation during DS- or SC-solidification was performed with a cellular automaton rotated grid algorithm. In this model the grain orientation is identified with the orientation of the grid. A detailed description of the rotated grid algorithm is given in [15]. 2D-calculations of grain selection development were performed. 3D-calculations are only done for the single crystal solidification. The grain growth of a single crystal in a simplified geometry of a turbine blade inner shroud was calculated, Figure 12. The results of the grain growth calculation with a grain orientation slightly rotated against the shrouds coordinate system (Euler angles $\vartheta=0^{\circ}, \psi=0^{\circ}, \varphi=8^{\circ}$ ) are shown in Figure 13. A transversal and two longitudinal ( $x$ - and $y$-plane) sections through the geometry at the same time step are displayed. The transversal section shows the slight rotation of the grain envelope which coincides with the crystallographic rotation. The longitudinal sections through the $x-$ and $y$-plane look the same due to the crystal's symmetry. The same situation was calculated with the 3D-CA coupled with FEM temperature field simulation, Figure 14. Again a transversal and two longitudinal sections through this geometry at the same time step are shown. These pictures clarify the influence of the calculated temperature field on the grain envelope development. The anisotropic growth into the edges of the shroud is due to the faster cooling in the center of the cluster. The curvature of the surface in the longitudinal sections is caused by the macroscopic curvature of the isotherm in the temperature field simulation [11].

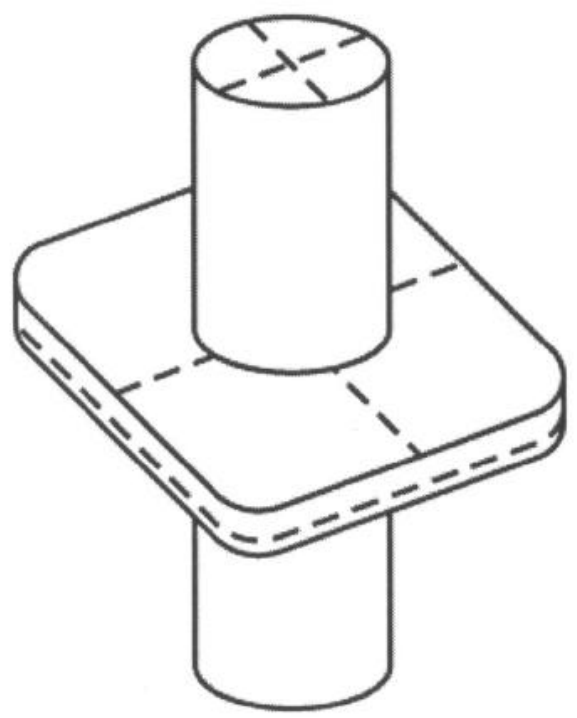

Figure 12: Principle sketch of the investigated simplified geometry of a turbine blade inner shroud. The lines indicate the sectional planes.

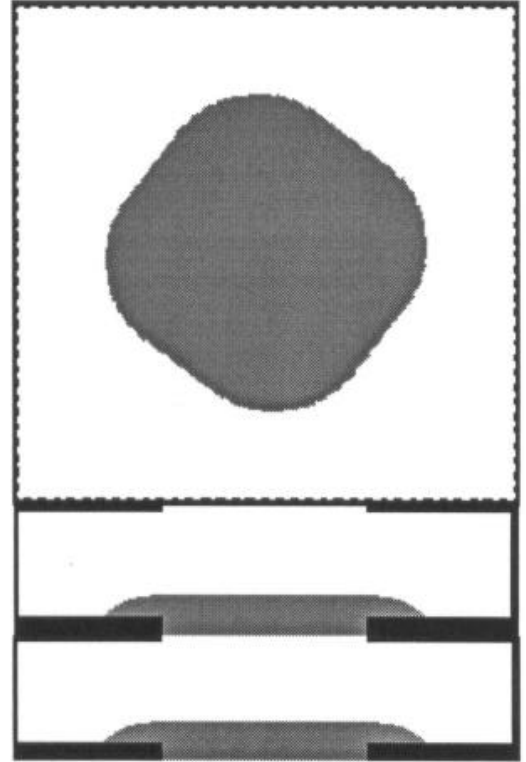

Figure 13: Transversal (upper picture) and longitudinal sections through the simplified geometry of an inner shroud. The gray areas represent the grain envelope of the single crystal whereas the white areas are still liquid. The snapshots were taken at the same calculation time step.

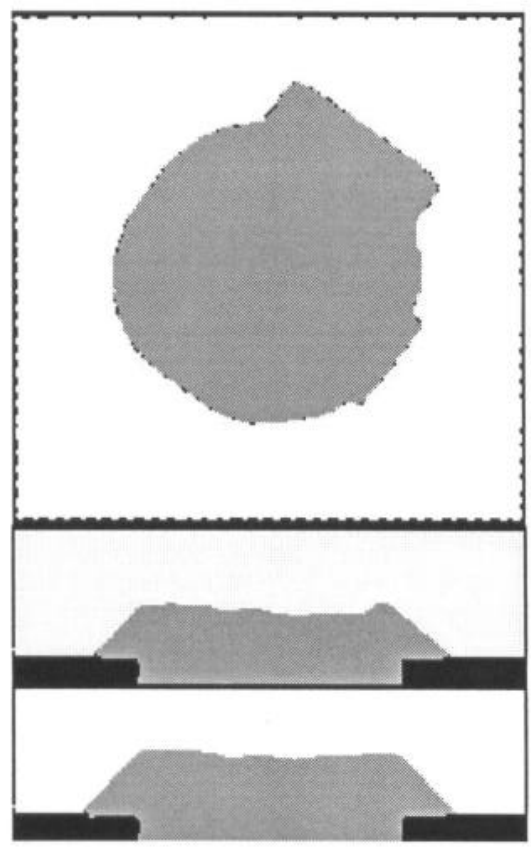

Figure 14: The same situation as in Figure 13 calculated with a $3 \mathrm{D}-\mathrm{CA}$ coupled with FEM temperature field simulation. The anisotropic growth into the edges of the shroud is due to the faster cooling in the center of the cluster. The curvature of the surface in the longitudinal sections is caused by the macroscopic curvature of the isotherm in the temperature field simulation. The snapshots were taken at the same calculation time step. 
To use this model for the prediction of freckling it will be necessary to include a fluid flow calculation and a nucleation model. This model has to consider undercooling as well as crystallographic and convection effects.

\section{Conclusions}

The main conclusions of the present work are as follows:

- A simple $G^{*} v$-criterion for practical use has been developed which takes into account varying local solidification conditions near solidus temperature during Bridgman process.

- FEM temperature field simulation combined with the experimental $G^{*} v$-criterion allows the prediction of component areas which are extremely freckle threatened and those areas, which are certainly freckle free.

- At solidification conditions typical of industrial production sized Bridgman furnaces freckles does not form inside the casting even at abrupt cross-scction cxpansions.

- By now it is not possible to predict where freckle formation actually will take place. An improvement in freckle prediction by using a combination of FEM-simulation including fluid flow models with cellular automaton calculations is part of further investigation.

\section{Acknowledgements}

The authors would like to thank $A$. Schievenbusch, ACCESS e.V., and G. Nolze, BAM, for performing the EDX- respectively EBSD- Analysis. Partially the project subject matter of the present report has been promoted by funds of the German Federal Ministry for Education, Science, Research and Technology under the promotion reference $0327058 \mathrm{C}$. Responsibility for the content of this publication lies with the authors.

\section{$\underline{\text { References }}$}

1. A.F. Giamei, B.H. Kear, "On the Nature of Freckles in Nickel Base Superalloys", Met. Trans., 1 (1970) 2185-2192

2. S.M. Copley et al., "The Origin of Freckles in Unidirectionally Solidified Castings", Met. Trans., 1 (1970), 2193 2203

3. T.M. Pollock, W.H. Murphy, "The Breakdown of SingleCrystal Solidification in High Refractory Nickel-Base Alloys", Met. and Mat. Trans. A, 27A (1996) 1081-1094

4. J.R. Sarazin, A. Hellawell, "Channel flow in partly solidified alloy systems", Advances in Phase Transition, 10 (1987), 101-115
5. P. Auburtin, S.L. Cockroft, A. Mitchell, Freckle Formution in Large Superalloy Single Crystal Airfoil Castings", Materials for Advanced Power Engineering, eds. J. Lecomte- Beckers, F. Schubert and P.J. Ennis, (FZ Jülich, 1998), 1459-1468

6. G.L. Erickson, "The development and application of CMSX $^{\circledR}-10^{\prime \prime}$, Supcralloys, eds. R.D. Kissinger, D.J. Deye, D.L. Anton, A.D. Cetel, M.V. Nathal, T.M. Pollock, D.A. Woodford, (TMS, 1996), 35-44

7. P.R. Sahm, W. Richter and F. Hediger, "Das rechnerische Simulieren und Modellieren von Erstarrungsvorgängen bei Formguß", Gießerei-Forschung, 35 (2) (1983), 35-42

8. U. Reske et al., "Numerische Simulation der gerichteten Erstarrung nach dem Bridgman-Verfahren", GießereiForschung, 43 (3) (1991), 101-106

9. N. Hofmann et al., "Numerische Simulation der gerichteten Erstarrung nach dem Bridgman-Verfahren II", Gießerei-Forschung_44 (3) (1992), 113-120

10. F. Hediger, N. Hofmann, "Process Simulation for Directionally Solidified Turbine Blades of Complex Shape", Modeling of Casting. Welding and Advanced Solidification Processes, eds. M. Rappaz, M.R. Özgü, K.W. Mahin, (TMS, 1991), 611-619

11. J.L.L. Rezende, "Numerical Modelling of Microstructure Formation during Directional Solidification utilizing the Bridgman Process" (Dissertation, RWTH-Aachen, 1999)

12. M.C. Schneider et al., "Modeling of Micro- and Macrosegregation and Freckle Formation in Single-Crystal Nickel-Base Superalloy Directional Solidification", Met. and Mat. Trans A, 28A (1997), 1517-1531,

\section{C. Beckermann, private communication}

14. S.D. Felicelli, D.R. Poirier and J.C. Heinrich, "Modeling Freckle Formation in Three Dimensions during Solidification of Multicomponent Alloys", Met. and Mat. Trans. B, 29B (1998), 847-855

15. N. Warnken et al., "Simulating Grain Structure Formation with a Rotated-Grid Cellular Automaton Algorithm", acceptcd for publication in, Modeling of Casting, Welding and Advanced Solidification Processes IX, eds. P.R. Sahm, P.N. Hansen, J.G. Conley, (2000) 